\title{
Short-Pulse Laser Materials Processing
}

\author{
B. C. Stuart, M. D. Perry, B. R. Myers, \\ P.S. Banks, and E. C. Honea
}

June 18, 1997

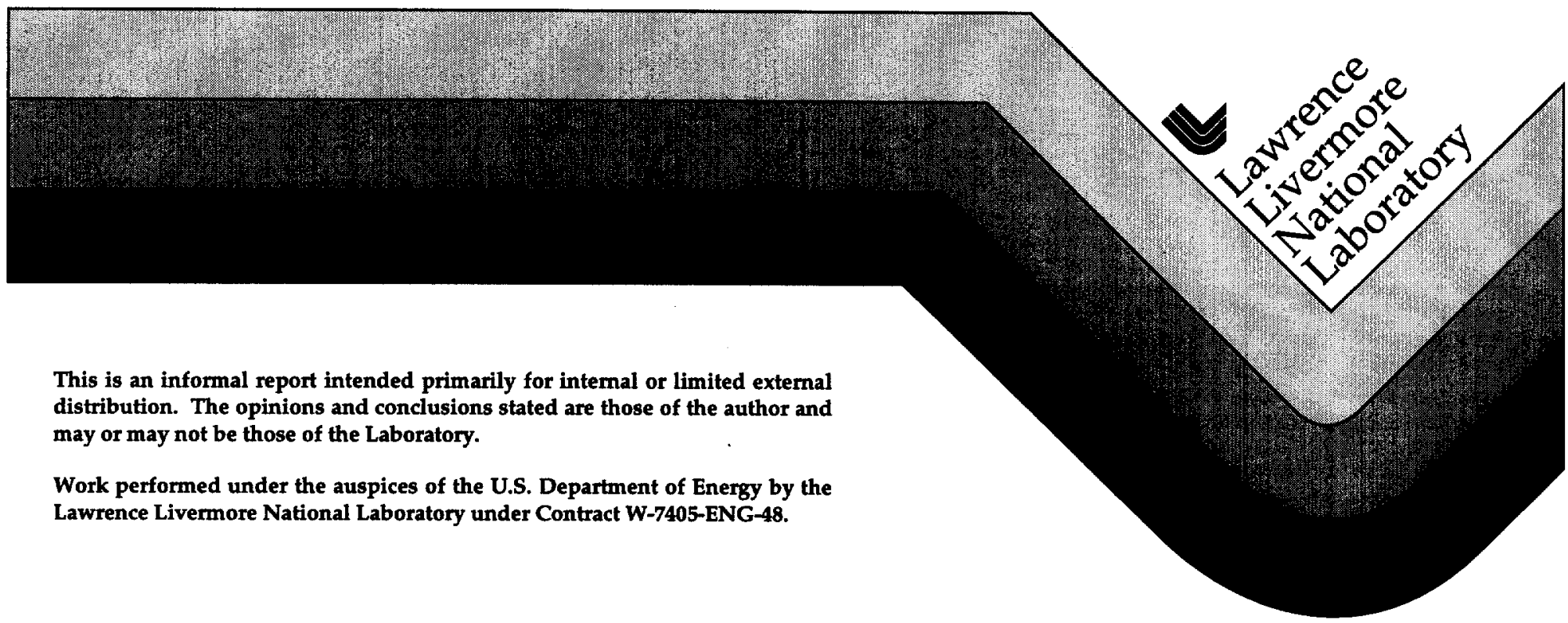




\section{DISCLAIMER}

This document was prepared as an account of work sponsored by an agency of the United States Government. Neither the United States Government nor the University of California nor any of their employees, makes any warranty, express or implied, or assumes any legal liability or responsibility for the accuracy, completeness, or usefulness of any information, apparatus, product, or process disclosed, or represents that its use would not infringe privately owned rights. Reference herein to any specific commercial product, process, or service by trade name, trademark, manufacturer, or otherwise, does not necessarily constitute or imply its endorsement, recommendation, or favoring by the United States Government or the University of California. The views and opinions of authors expressed herein do not necessarily state or reflect those of the United States Government or the University of California, and shall not be used for advertising or product endorsement purposes.

This report has been reproduced directly from the best available copy.

Available to DOE and DOE contractors from the Office of Scientific and Technical Information

P.O. Box 62, Oak Ridge, TN 37831

Prices available from (615) 576-8401, FTS 626-8401

Available to the public from the

National Technical Information Service

U.S. Department of Commerce

5285 Port Royal Rd.,

Springfield, VA 22161 


\title{
Short-Pulse Laser Materials Processing
}

\author{
B. C. Stuart, M. D. Perry, B. R. Myers, P. S. Banks, E. C. Honea
}

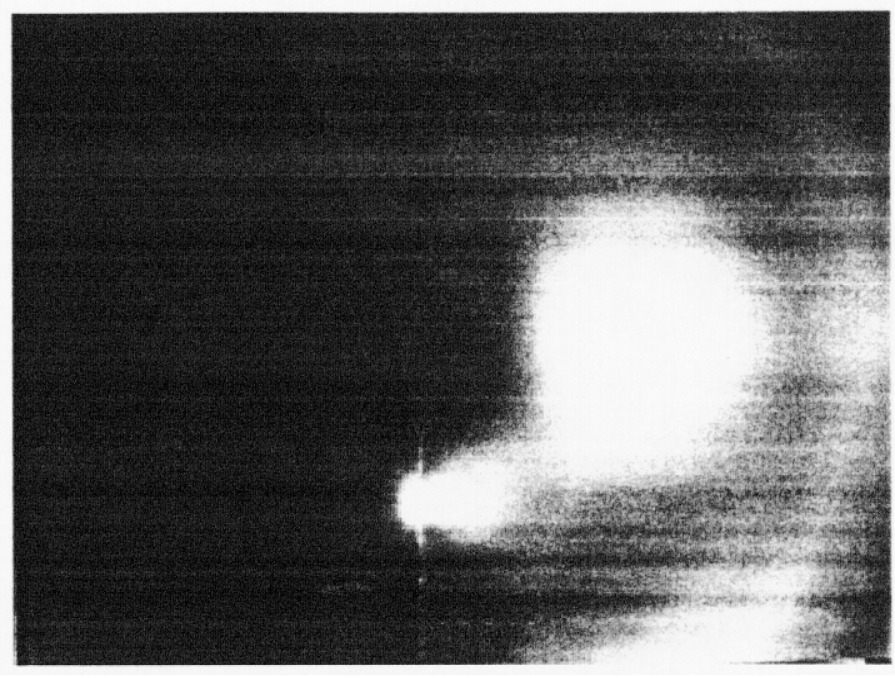

\section{Summary}

While there is much that we have learned about materials processing in the ultrashort-pulse regime (see background below), there is an enormous amount that we don't know. How short does the pulse have to be to achieve a particular cut (depth, material, quality)? How deep can you cut? What is the surface roughness? These questions are clearly dependent upon the properties of the material of interest along with the short-pulse interaction physics. From a technology standpoint, we are asked: Can you build a $100 \mathrm{~W}$ average power system? A $1000 \mathrm{~W}$ average power system?

The answers to these questions are critical to our ability to develop this technology into a viable industrial processing technology and to develop sponsors for this development at LLNL. This proposal seeks to address these questions with a combined experimental and theoretical program of study. Specifically,

1) To develop an empirical data base for both metals and dielectrics which can be used to determine the pulse duration and wavelength necessary to achieve a specified machining requirement.

2) To investigate $\mathrm{Yb}: \mathrm{YAG}$ as a potential laser material for high average power short-pulse systems both directly and in combination with titanium-doped sapphire. We will examine the pulse repetition rate and beam quality issues associated with this material both theoretically and experimentally using an end-pumped Yb:YAG amplifier constructed for another program. This effort will draw upon the expertise of many in the Laser Program (e.g., Steve Payne, Ray Beach, David Eimerl, and many others).

3) Develop a conceptual design for a $100 \mathrm{~W}$ and eventually $500 \mathrm{~W}$ average power short-pulse system. 


\section{Background}

Conventional mechanical lathes and machine tools (e.g., slitting saws) are effective for cutting metals down to approximately 100 microns kerf width at depths on the order of 1 millimeter (aspect ratio $<10: 1)$. Below this level, electron beam or laser tools are typically used for cutting or high precision machining (sculpting, drilling). Both electron beam and existing industrial laser technology remove material by a conventional thermal process where the material to be removed is heated to the melting or boiling point. Laser processing by molecular dissociation in organic (and some inorganic) materials can be achieved with excimer lasers but this photodissociation mechanism is not applicable to metals.

The basic interaction with conventional lasers is the deposition of energy from the incident beam in the material of interest in the form of heat (lattice vibrations). The laser energy that is absorbed results in a temperature increase at and near the absorption site. As the temperature increases to the melting or boiling point, material is removed by conventional melting or vaporization. Depending on the pulse duration of the laser and the thermomechanical properties of the material, the temperature rise in the irradiated zone may be very fast resulting in thermal ablation and shock. The irradiated zone may be vaporized or simply ablate off due to the fact that the local thermal stress has become larger than the yield strength of the material (thermal shock). In all these cases, where material is removed via a thermal mechanism there is an impact on the material surrounding the site where material has been removed. The surrounding material will have experienced a large temperature excursion or shock often resulting in significant change to the material properties. These changes may range from a change in grain structure to an actual change in composition. Such compositional changes include oxidation (if cut in air) or, in the case of alloys, changes in composition of the alloy. This effected zone may range from a few microns to several millimeters depending on the thermomechanical properties of the metal, laser pulse duration and other factors (e.g., active cooling). In many applications, the presence of the heat or shock effected zone may be severely limiting since the material properties of this zone may be quite different than that of the bulk. Furthermore, devices with features on the order of a few tens of microns cannot tolerate the thermal stress induced in the material during the machining process.

Another limitation of conventional laser processing in high precision applications is the presence of redeposited or resolidified material. The surface adjacent to the removed area will have experienced significant thermal loading often resulting in melting. This melting can be accompanied by flow prior to solidification. This can result in the deposition of slag surrounding the kerf (Figure 1). In many high precision applications, the presence of slag is unacceptable. In the cases where the deposition of conventional slag can be prevented, redeposition of vaporized material on the walls or upper surface of the kerf is common. This condensate often reduces the quality of the cut and decreases the cutting efficiency since the beam must again remove this condensate before interacting with the bulk material underneath.

Many of these limitations can be reduced by the use of secondary techniques to aid the cutting process. The most common of these are active cooling of the material of interest either during or immediately following the laser pulse, and the use of high pressure gas jets to remove vaporized or molten material from the vicinity of the cut to prevent redeposition. These techniques can be effective at improving the kerf at the cost of a significant increase in system complexity and often a decrease in cutting efficiency. 


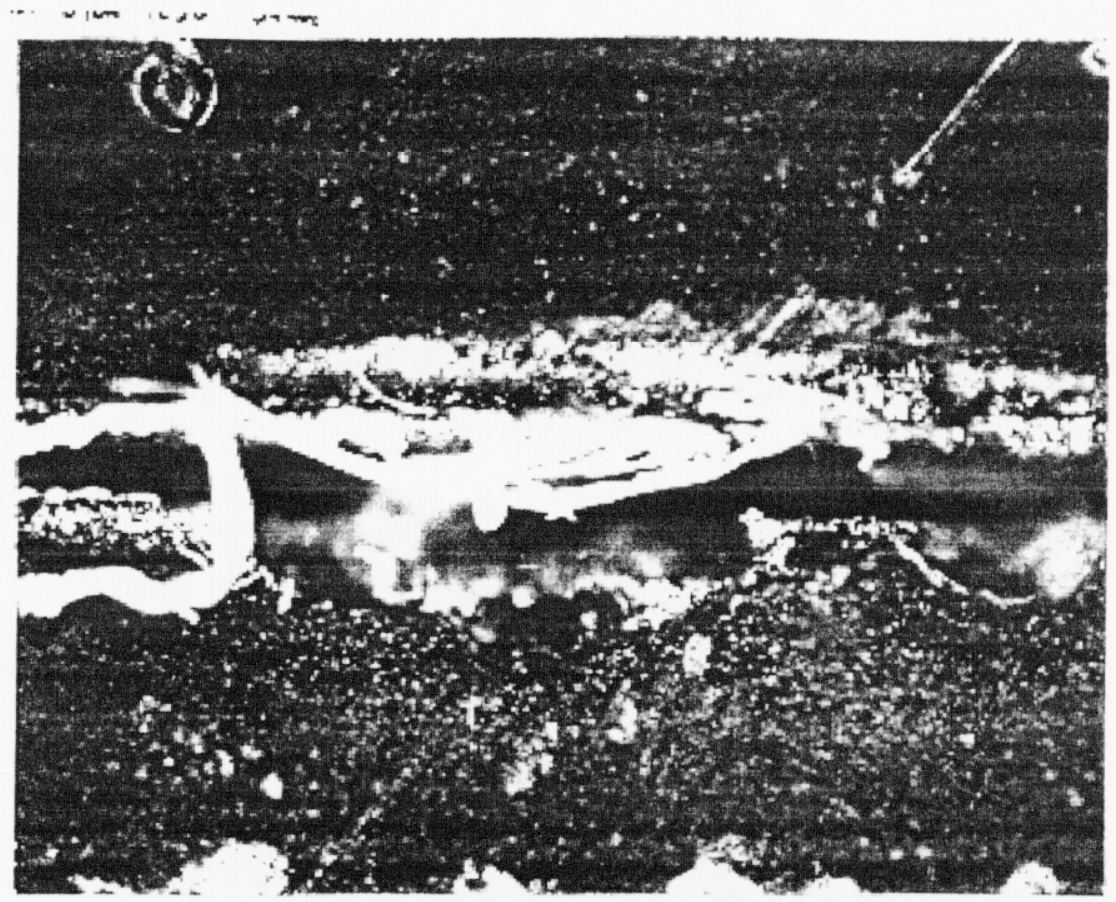

Figure 1a: Top View of Stainless Steel cut with a conventional $1053 \mathrm{~nm}$ laser operating at a pulse duration $>1 \mathrm{nsec}$. The presence of resolidified molten material (slag) and poor single pass cut quality indicative of laser cutting by conventional methods is readily apparent.

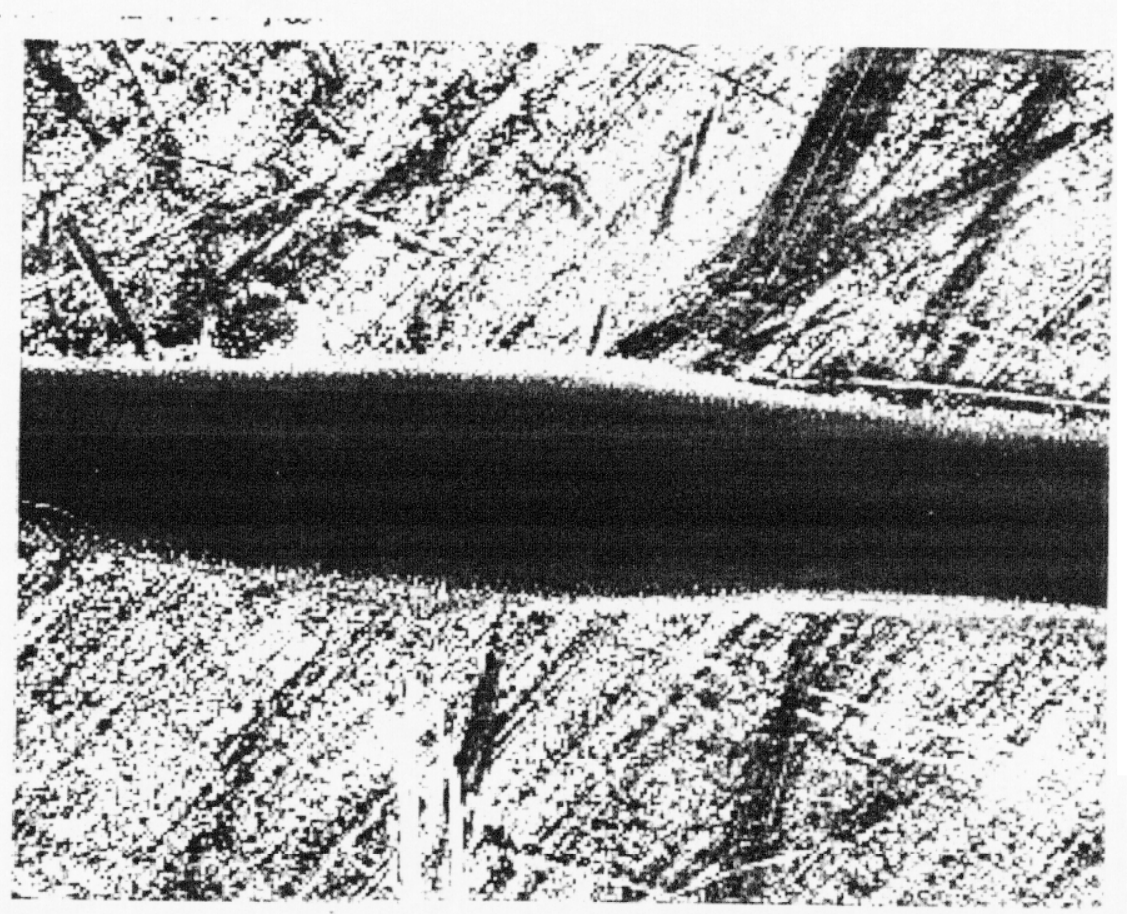

Figure 1b: Top View of Stainless Steel as in Figure 1a but cut with a $350 \mathrm{fsec}$ pulse. The fluence on the steel surface was $14 \mathrm{~J} / \mathrm{cm}^{2}$ corresponding to an irradiance of $4 \times 10^{13} \mathrm{~W} / \mathrm{cm}^{2}$. 
We have shown previously that by using femtosecond pulses laser machining of metals and alloys can be achieved with high speed, extreme precision, negligible heat affected zone, and no modification to the material surrounding the kerf. Although the absorption mechanism for the laser energy in metals is the same as in the case of long pulse lasers, the short duration offers an important advantage. By adjusting the pulse duration such that the thermal penetration depth during the pulse, $L_{t h}=$ $2 \sqrt{ } \alpha \tau$, is less than one micron, very small amounts of material $(0.01-1$ micron) can be removed per laser pulse with extremely small transport of energy either by shock or thermal conduction away from the volume of interest. This offers extremely high precision machining with no heat or shock effected zone. For example, type 304 stainless steel exhibits a thermal penetration depth of only $1.5 \mathrm{~nm}$ for a 100 femtosecond pulse compared to an optical penetration depth of approximately $5 \mathrm{~nm}$. The lack of significant energy deposition beyond the volume of interest achieved by using these ultrashort pulses enables the use of high repetition $(0.1-100 \mathrm{kHz})$ lasers without the need for external cooling of the part being machined. Even though only a very small depth of material is removed per pulse, the high repetition rate enables extremely high rates $(>1 \mathrm{~mm} / \mathrm{sec})$.

When cutting thick material at high aspect ratio (thin kerf in thick material, e.g., $50 \mu \mathrm{m}$ kerf in 1 $\mathrm{mm}$ thick steel), an irradiance beyond the saturation level is often required to achieve sufficiently high plasma temperature to prevent redeposition on the kerf. This is due to two factors. First, in high aspect ratio cutting, the kerf itself may function as a waveguide. This both reshapes the spatial distribution of the laser light and reduces the intensity reaching the bottom of the kerf. As a result, the fluence incident on the bottom of the kerf may be substantially less than that incident on the part surface. Second, as the plasma expands from the surface, it cools. The irradiance must be high enough at the bottom of the kerf to insure a high enough temperature such that when the plasma expands and cools, it cannot cool to the point where it can condense on the walls of the kerf as it exits. This high irradiance can be achieved either by shortening the pulse duration at a fixed fluence (Figure 2) or by increasing the fluence for a fixed duration.

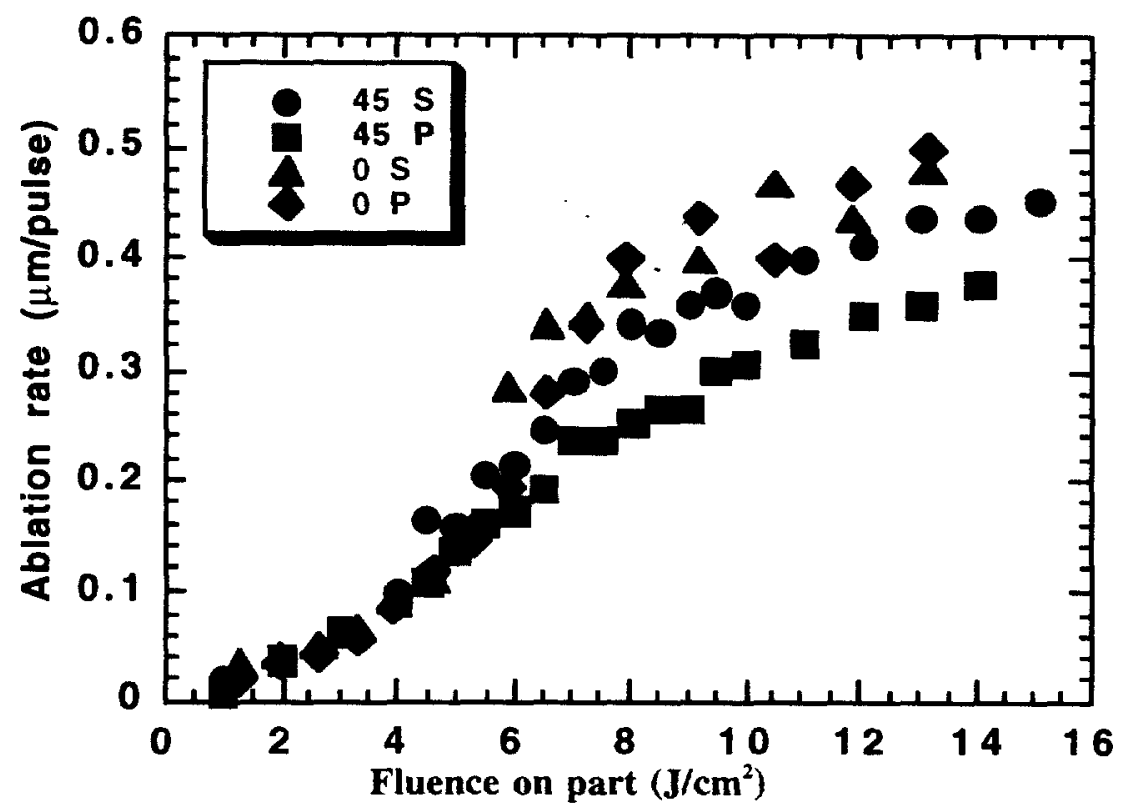

Figure 2: Depth of material removed per pulse as a function of laser fluence for 120 fsec pulses. The data was taken in the limit of no waveguide effects $(140 \mu \mathrm{m}$ thick steel). 


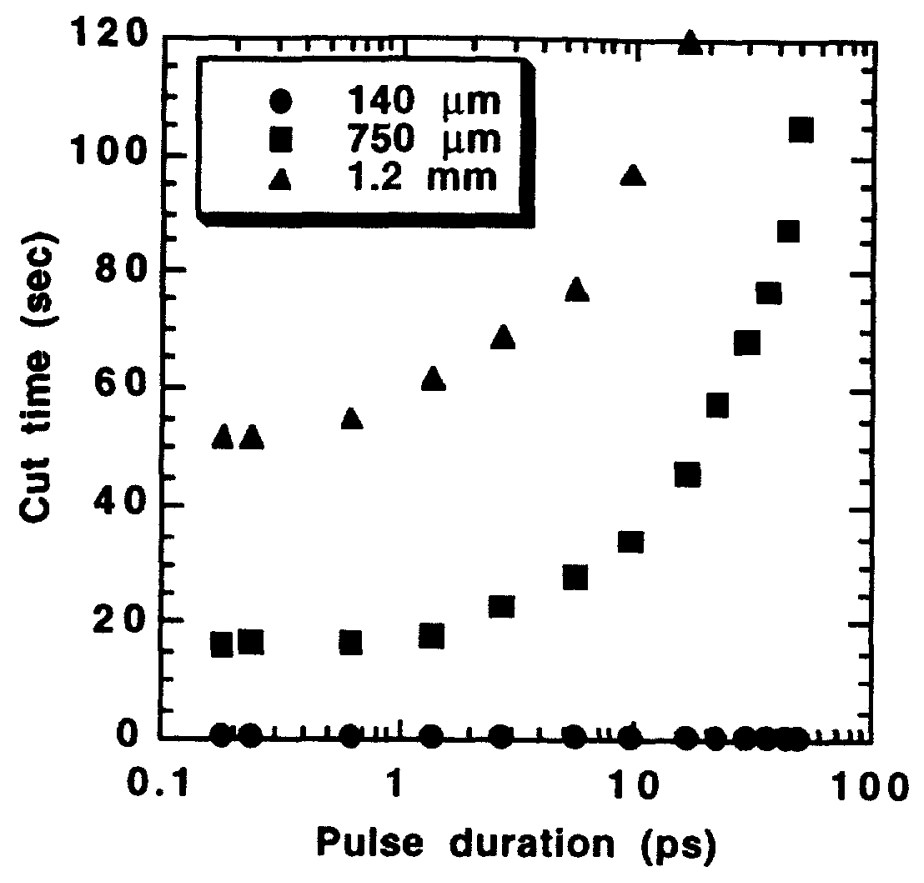

Figure 3: Time required to cut through stainless steel of various thickness as a function of pulse duration at a fixed fluence of $12 \mathrm{~J} / \mathrm{cm}^{2}$. The pulse repetition rate of the laser was $1 \mathrm{kHz}$.

\section{Dielectrics}

In the case of dielectric materials (ceramics, teeth, bone, heart tissue, etc.), cutting and drilling is achieved by a fundamentally different mechanism'. With femtosecond pulses, laser energy is initially absorbed by multiphoton ionization. This process creates free electrons in the dielectric which are rapidly accelerated by the electric field of the laser. These electrons create further free electrons by ionizing collisions with adjacent atoms. With pulses of a duration much shorter than the energy transfer time to the lattice ( 10 ps for most dielectrics), this process creates a high density plasma with negligible energy deposition beyond the thin plasma layer on the surface. This region is characterized by a clear deviation from the nominal $\tau^{1 / 2}$ dependence of damage fluence on pulse duration (Figure 4). There is no thermal shock to the surrounding material since the lattice remains cold. 


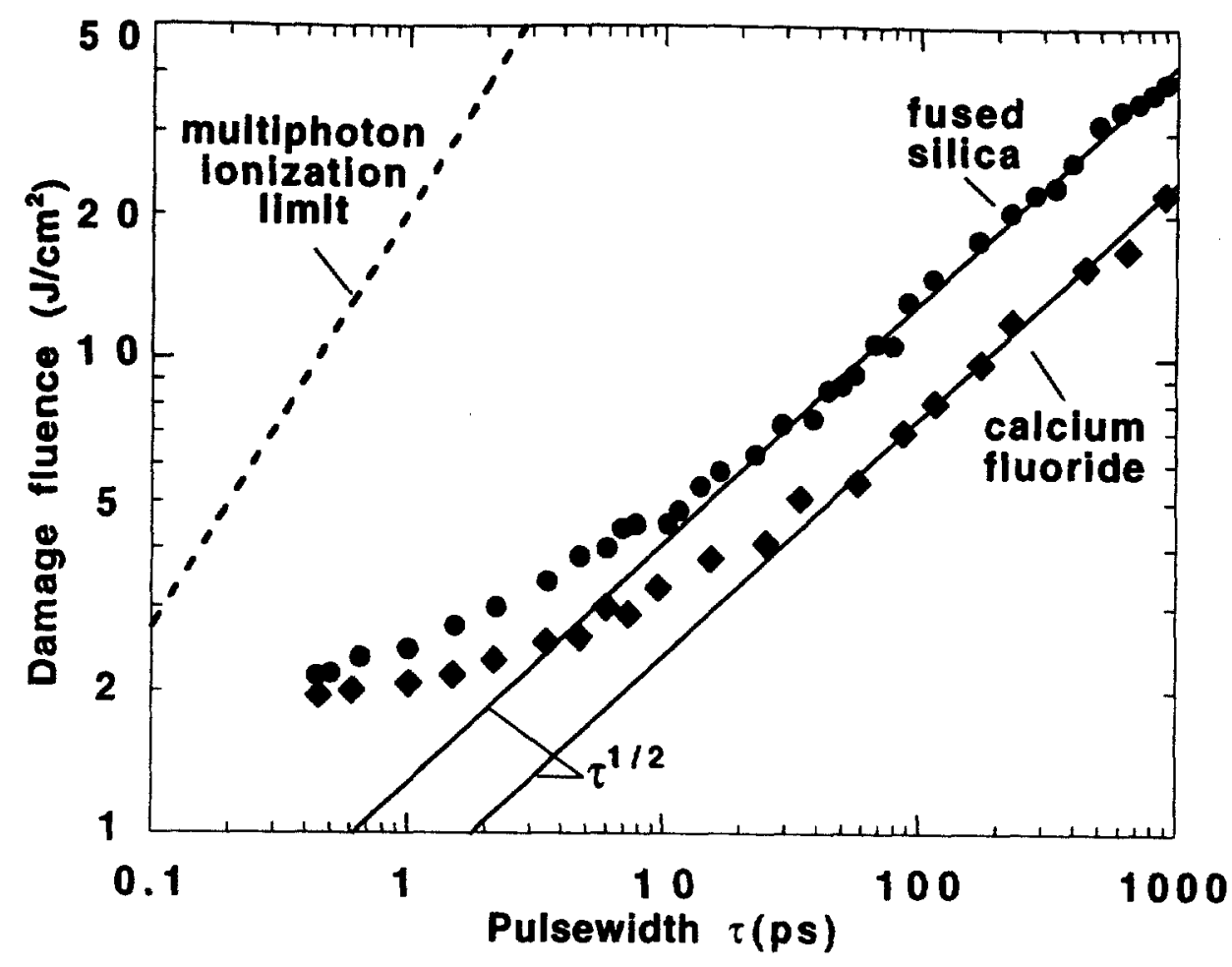

Figure 4: Threshold for material removal as a function of pulse length for a $1054 \mathrm{~nm}$ laser. The $\tau^{1 / 2}$ scaling for pulses beyond $10 \mathrm{ps}$ is characteristic of a thermal mechanism and is well established prior to this work.

The precision with which material can be removed is unprecedented due to the highly nonlinear nature of the multiphoton initiation step. There features are easily demonstrated in Figure 5 which shows the onset of material removal from a fused silica grating surface. In this electron micrograph, small ridges $(0.22 \mu \mathrm{m}$ width) exhibit material removal from only one edge while the other remains unaffected. Shown in Figure 5b, is the corresponding electric field distribution within the grating structure. Only the edge which exhibited material removal experiences a high field, the other edge only $0.2 \mu \mathrm{m}$ always sees a relatively low laser field and remains unaffected. By changing the pulse duration to be in the conventional regime, we observe the destruction of the entire grating surface and several layers below due to thermal shock. 


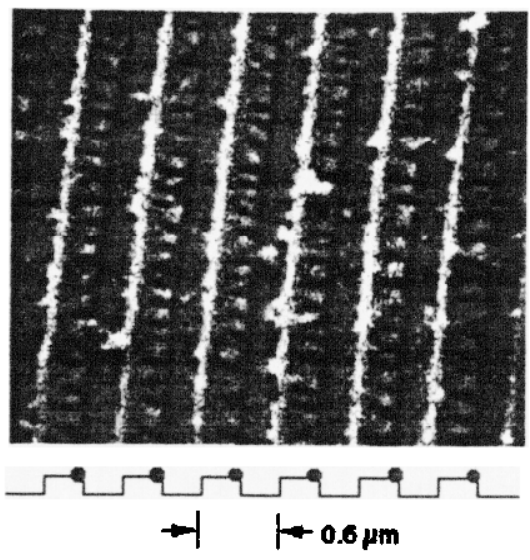

Figure 5a: Initial ablation of grating grooves when irradiated by 100 -fs pulses. Material is removed only from the regions of high electric field strength.

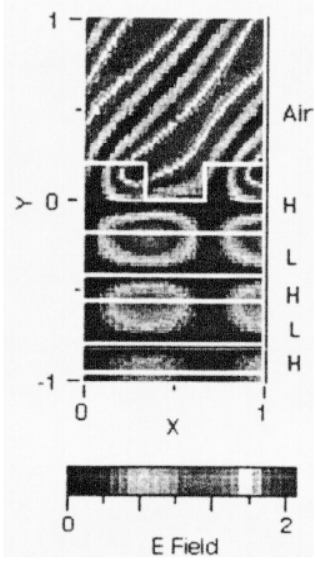

Figure 5b: Theoretical electric field distribution at interface of multilayer dielectric grating. The field is enhanced along one edge of each ridge.

On a macroscopic scale, the nonthermal nature of material removal from dielectric is manifest as extraordinary clean kerfs with no cracking or temperature rise in the bulk. In the case of conventional laser pulses, laser the enamel must be heated to the boiling point while the bulk of the tooth remains relatively cold. This results in thermal shock and brittle failure of the enamel similar to a hot glass placed under cold water. In the short-pulse regime, the absence of thermal shock and the plasma removal mechanism combine to produce extremely clean drill holes with no cracking. In Figure 6, we show the temperature rise of the bulk tooth as a function of drilling time. With conventional laser pulses, the bulk tooth increases in temperature by $>50^{\circ} \mathrm{C}$ while with fsec pulses, the temperature rise is less than $2^{\circ} \mathrm{C}$.

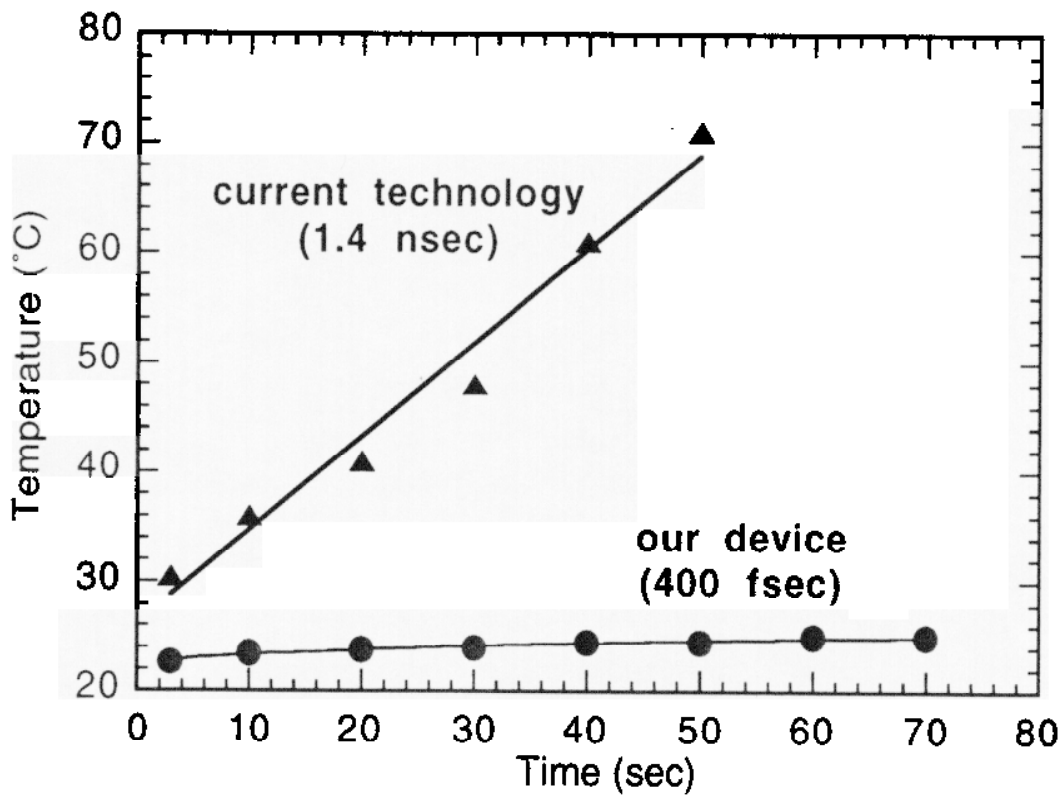

Figure 6: Temperature $1 \mathrm{~mm}$ from ablation site associated with enamel drilling with current technology (triangles) and with our device (circles). An increase of $5{ }^{\circ} \mathrm{C}$ will kill the root. In both cases, the laser wavelength was $1054 \mathrm{~nm}$ and the material removal rate was $1 \mu \mathrm{m} /$ pulse at $10 \mathrm{~Hz}$. 


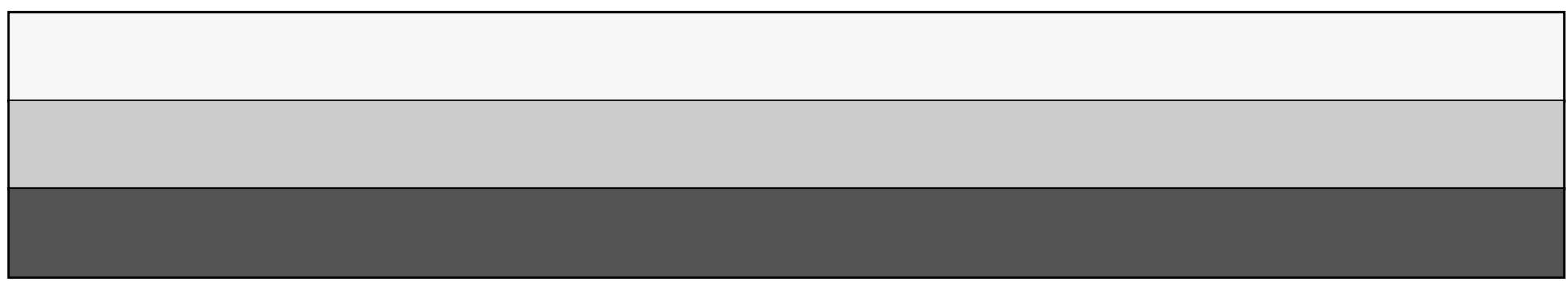

